\title{
Flow past porous cylinder/sphere with/without blowing
}

\author{
Hsing-Sheng Chai ${ }^{1 *}$, Hui-Zhen Chen ${ }^{2}$, Chiun-Hsun Chen ${ }^{3}$ \\ ${ }^{1}$ General Education Center, Aletheia University, Taipei, Taiwan \\ 2, 3 Department of Mechanical Engineering, National Chiao Tung University, [Hsinchu, Taiwan
}

\section{Keywords \\ Porous circular cylinder Porous sphere \\ Blowing}

Received: 27 February 2018

Accepted: 21 March 2018

Published: 18 April 2018

\begin{abstract}
Flow characteristics around/behind a porous circular cylinder and porous sphere in a wind tunnel are studied. The parameters are incoming flow velocity $\left(U_{i n}\right)$, air ejection velocity $\left(V_{w}\right)$ from the surface of cylinder/sphere, and dimension/shape of cylinder/sphere. This study is divided into dual parts. First part deals with flow field with no air ejection from the surface of cylinder/sphere, and the second one deals with the flow field for air ejection from cylinder/sphere surface. Finally, for each cylinder/sphere, $V_{w}$ and critical $U_{i n}$ that causes the wake returns to the rear of cylinder/sphere are correlated into equations, respectively.
\end{abstract}

(C) 2018 The Author(s). Published by TAF Publishing.

\section{INTRODUCTION}

This study is motivated from work of [1, 2]. He found that a lift-off flame occurs after a wake flame as the incoming flow velocity further increases, then it jumps back in the form of wake flame again when the velocity continues to increase. [3] experimentally investigated the lift-off flame, too. They indicated that the lift-off flame arises because the high pressures generated at the two flame fronts depress the vortices behind the cylinder and destroy them eventually. When the lift-off height is large enough, the vortices are generated again behind the cylinder. Then, the somewhat unstable lift-off flame front is drawn back by the recirculating flow to become a wake flame again. However, no influence of flow structures, which were interacted with the combustion and vice versa, could be visually observed in the flow field due to the existence of flame. Therefore, the present work intends to investigate the cold flow field around and behind the cylinder/sphere to understand their influence on the resultant flames. The experimental set-up basically is the same as the one used by [3], except the additions of the smoke-generation wire and laser-sheet lighting, as shown in Figure 1.

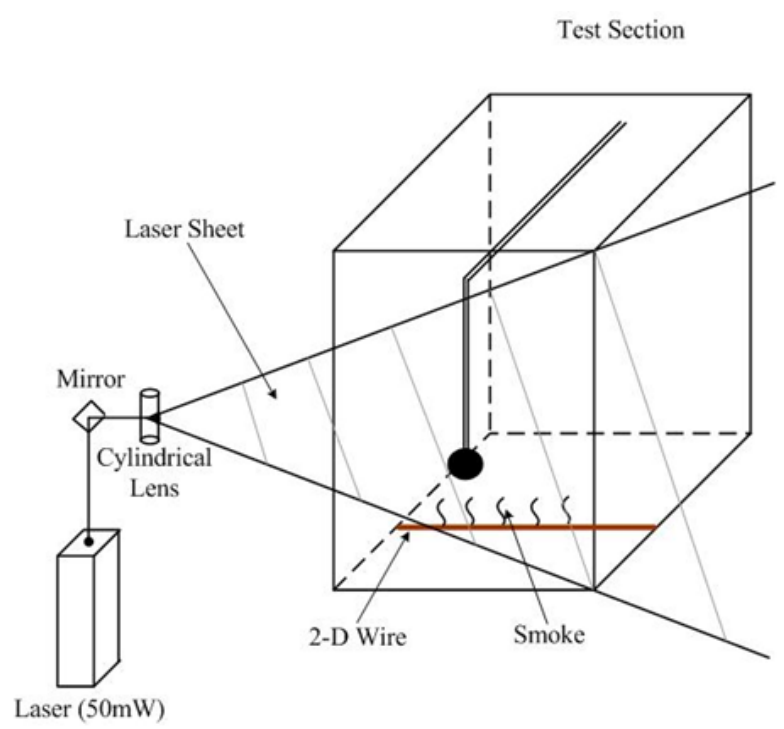

Fig. 1. Schematic configuration of smoke-generation wire and laser-sheet lighting

The cold flow field visualization carried out in this study has dual parts. The first one is to investigate the flow field around and behind the cylinder and sphere without air blowing. In the second part, the air is used and ejected

\footnotetext{
${ }^{*}$ Corresponding author: Hsing-Sheng Chai

†email: au4289@mail.au.edu.tw
} 
from the cylinder and sphere into the incoming air flow. The porous cylinder used in this study has modified from the original design of $[3,4]$ but without cooling groove. The significance of this study is to identify the influences of incoming flow velocity $\left(U_{i n}\right)$, air ejection velocity $\left(V_{w}\right)$, cylinder diameter, and geometric variations of cylinder/sphere on the flow field around the circular cylinder/sphere. Investigating the effects of these four parameters simultaneously in flow characteristics around and behind the porous circular cylinder/sphere are the spotlights. Besides, the results of the flow visualization are described in detail. The resultant photographs will be introduced to comprehend the flame behaviors for the cylindrical burners by the influence of flow structures. In particular, the physical mechanisms of the lift-off flame and the second wake flame are identified more clearly. Finally, the flow patterns taken by a digital video are used to measure the separation angle that originated from the front stagnation point to the separation point.

\section{LITERATURE REVIEW}

[5] visualized the wake flow behind a sphere. He found that separation from the sphere starts to occur at $R e \sim 24$ and results in the generation of a symmetric vortex ring. Regarding the stability of the resultant wake, he observed a faint periodic motion 'with a very long period' at the rear of vortex ring beginning at $R e=130$. [6] investigated the stability of the symmetric sphere flow using a finite-element method. They found a regular bifurcation, namely, a transition to steady flow, at $R e=210$ and naturally suggested that this corresponds to a transition from the steady, symmetric wake to the steady, asymmetric, and double-thread wake. The observed onset of the shedding regime covered the range of $290<R e<400$. [7] and [8] presented the frequency measurements for unsteady wake. As Re was close to 400 or up to 420 , the onset of unsteadiness, a strongly periodic process, was identified by the observation of a single frequency in the wake at a Strouhal number (St) in the range of $0.15<$ St $<0.17$. The numerical solutions of the unsteady flow past a sphere was carried out by [9]. They recorded that the unsteady transition was in the range of $270<R e<$ 285. [10] numerically and experimentally investigated an incompressible viscous fluid flow past a sphere. Their calculations for Re up to 200 showed a steady, symmetric flow and they agreed well with the previous experimental observations. For $R e$ from 210 to 270 , a steady, asymmetric flow was found and it was also in agreement with the previous work. Unsteady flow was predicted at Re greater than 270 . [11] utilized numerical simulation to perform the down- stream wake control behind a cylinder. They studied the effect of location change of discharge electrode and the discharge electrode radius on the reduction of downstream wake behind a circular cylinder. The results showed that the corona wind can't diverge the main stream into a circular cylinder at high Reynolds numbers. [12] experimentally investigated the wake flow behind cylinders with different taper ratios at numerous sub-critical Reynolds numbers. The taper ratios used were ranged from 0.6 to 1 with an increment of 0.1. Parameters that they investigated were wind speed ratios, wake width, and dissipation length at each taper ratio. [13] numerically investigated the use of flags to decline drag surrounding a cylinder. An unsteady turbulent simulation procedure was adopted at $R e=20000$. The results showed that the pressure drag was declined with the use of flags inside the near-wake zone of the circular cylinder.

Flow characteristics behind a circular cylinder were measured and visualized by using a time-resolved particle image velocimetry (TR-PIV) by [14]. The incoming flow velocities they utilized were 5 and $10 \mathrm{~m} / \mathrm{sec}$, respectively. Detailed flow structures were captured in a low Re number wind tunnel, and the cross section of their wind tunnel was $30 \times 30 \mathrm{~cm}^{2}$. [15] adopted 2D unsteady Computational Fluid Dynamics (CFD) model to estimate the drag and lift around a circular cylinder at $R e=189$. Turbulence model he utilized was DNS model. The sensitivities and fluctuations of drag and lift were calculated, and further applications were also discussed. For the applications of civil engineering, [16] made a detailed review for all types of so-called "circular cylinder" flow characteristics. The irregularities of cylinder were discussed in detail, and the acting forces that acted on the cylinder were also elucidated. Good collections and comparisons at circular cylinder flow field applications were revealed. The flow behavior over a modified circular cylinder was investigated experimentally by [17]. A streamwise slit that paralleled to the inflow air was adopted to measure its influence on pressure distributions and aerodynamic forces. The effects of slit width were studied, and the flip-flop characteristics were found, too.

\section{EXPERIMENTAL APPARATUS}

Basically, the experimental setup consists of a wind tunnel and porous sintered sphere/cylinder. In particular, the applications of the smoke-generation wire and the laser-sheet lighting are used to visualize the cold flow field around and behind the cylinder/sphere. Figure 2 depicts the entire experimental structure. The apparatus are described as follows: 


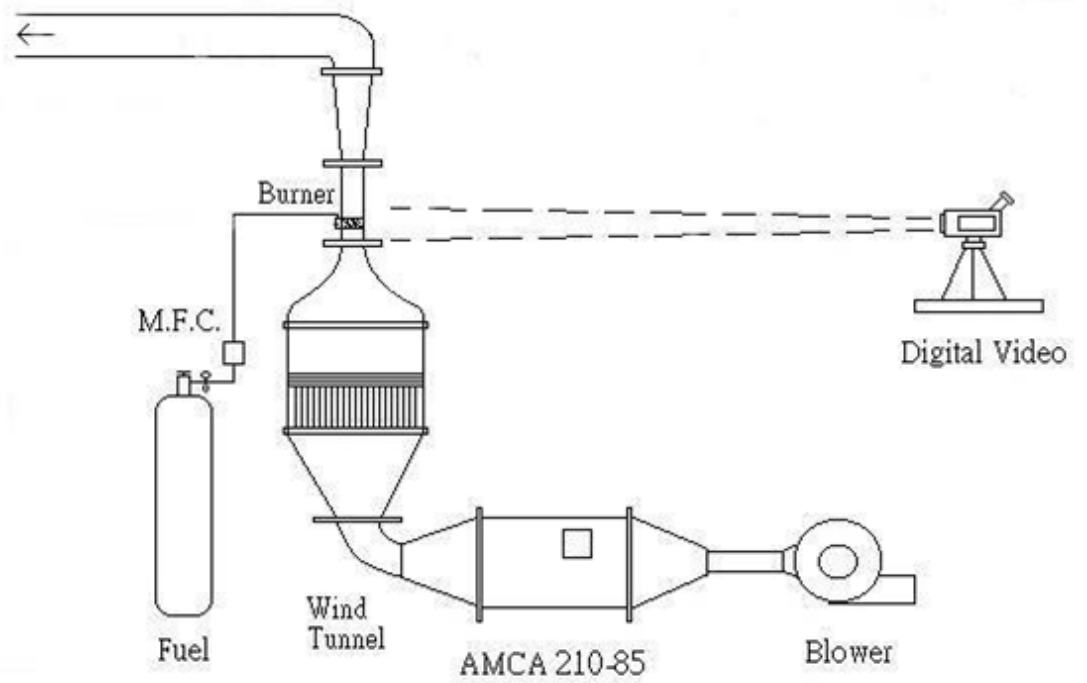

Fig. 2. Schematic drawing of overall experimental system

\section{A. Wind Tunnel}

The wind tunnel is open-circuit and orientated vertically upwards. The wind tunnel consists of five components, which are the blower, diffuser, flow straightener, contraction, and test section. Most of the design concepts are mainly from NIST [18]. Inlet velocity of test section is determined by AMCA 210-85 standard nozzle-method: the volume flow rate is determined and used to deduce the flow velocity. The error in AMCA $210-85$ is within $3 \%$ when the velocity is between $0.21 \mathrm{~m} / \mathrm{sec}$ and $3.3 \mathrm{~m} / \mathrm{sec}$, but becomes $5 \%$ when the velocity is under $0.21 \mathrm{~m} / \mathrm{sec}$.

\section{B. Blower}

The airflow in the tunnel is supplied by a variable-frequency blower, in which frequency is directly converted into desired velocity by converter. In order to avoid the influence of vibration, the bases of wind tunnel and blower are separated.

\section{Diffuser}

A 30-cm long diffuser has an inlet cross-section area of $12 \times 12 \mathrm{~cm}^{2}$ and the outlet one is $40 \times 40 \mathrm{~cm}^{2}$. The expansion ratio based on area is 1:11.

\section{Flow Straightener}

The inflow from the diffuser is unstable before entering the contracting section. The purpose of flow straightener is to modulate the inflow to become more stable. It consists of honeycomb and screen.

\section{E. Contraction Section}

The design of contraction section is required to shorten the duct and reduce boundary layer thickness along the wall as possible. Its purpose is to provide a uniform flow field into the test section.

\section{F. Test Section}

The test section has a cross section area of $24 \times 4 \mathrm{~cm}^{2}$ and a length of $30 \mathrm{~cm}$. It is made of four sides. In the front and two adjacent sides, they are equipped with quartz-glass plates as the observation windows.

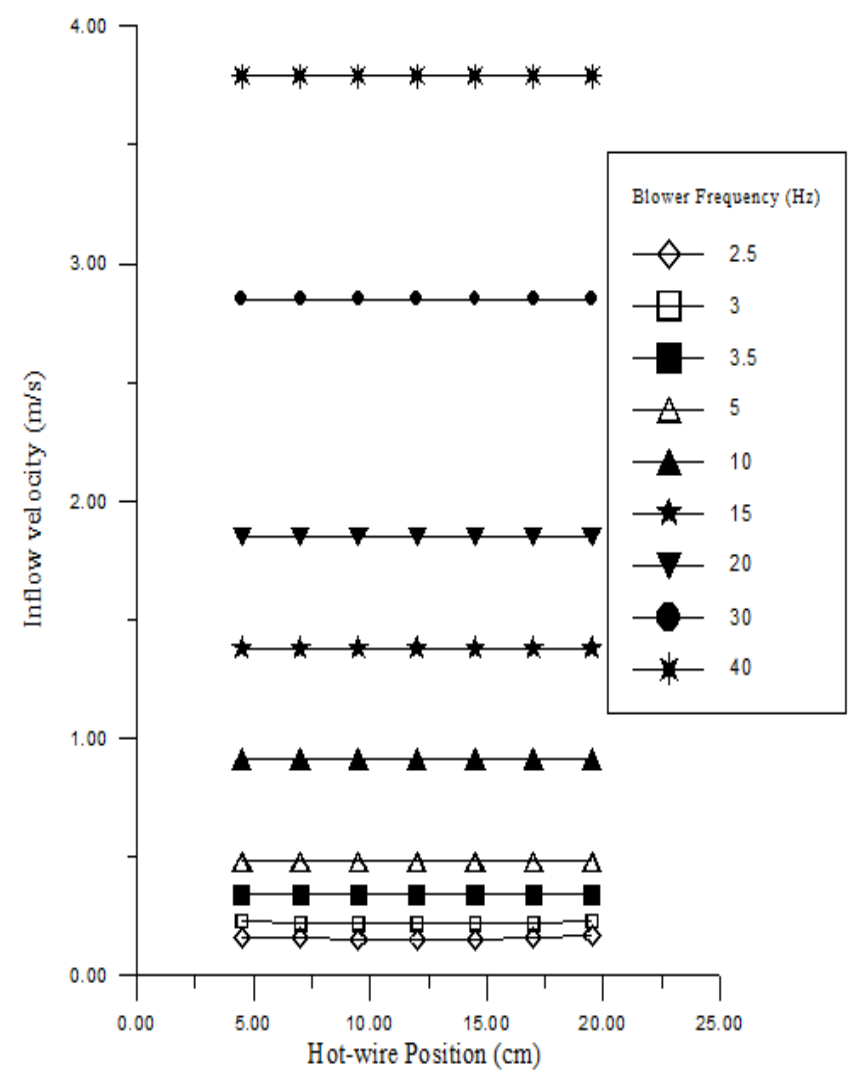

Fig. 3. Inflow velocity at each position in the test section 
The downstream of test section is connected to a diffuser (50 cm long), which can reduce the velocity of exhaust gases from test section. The vent follows after the diffuser to outdoor. At the rim of test-section entrance, there are seven holes to let sensors plug into flow to confirm its uniformity. The sensors are hot wires. If the velocities at each position are closely equal, then it can be certain that the flow is uniform and stable ahead of the burner, as shown in Figure 3.

\section{G. Porous Sintered Cylinder/Sphere}

The porous cylinder is comprised of inner and outer parts. In the outer part, it is a replaceable piece of porous sintered stainless steel, with $20 \mu \mathrm{m}$ pores and a length of $40 \pm 0.5$ $\mathrm{mm}$. The inner and outer diameters for one of the cylinders are $14 \mathrm{~mm}$ and $20 \mathrm{~mm}$, respectively. Nevertheless, the inner and outer diameters for the other one are $9 \mathrm{~mm}$ and 15 $\mathrm{mm}$, respectively. The inner part of the cylinder is a cylindrical brass rod with air supply groove. The outer part is screwed onto the inner part.

The outer diameter of the porous sphere is $15 \mathrm{~mm}$. The sphere is dug a fillister in order to connect to the gas transport line and the diameter and the depth of the fillister are $6.35 \mathrm{~mm}$ and $9.8 \mathrm{~mm}$, respectively. The transport line is in horizontal orientation across the stream.

The air is ejected from the surface of cylinder/sphere, and its flow rate is controlled and measured using a digital mass flow controller (MC-2100ENC, Lintec), with a highperformance microprocessor. The air ejection velocity is computed by dividing the air volumetric flow by the available air ejection area along cylinder/sphere surface.

\section{H. Smoke-Wire Technique}

In the present experiment, a solution of organic oil in water is adopted to generate the smokes. The diameter of wire is $0.1 \mathrm{~mm}$ and the wire is made of stainless steel. The fine wire is heated to vapor the oil by means of an electric current. The wire is coated uniformly with the oil to make certain that the smoke is produced evenly along the length of the wire. A thin sheet of light can be generated easily by using a cylindrical lens and a light source, like a laser. The power of laser is $50 \mathrm{mw}$, and it generates green light. The set-up position of laser is on the side of test section to visualize the smoke flow from the obverse side, and the height is changed arbitrarily from upstream of the test section. The schematic configuration is shown in Figure 1.

\section{Visualization System}

A digital video camera (SONY DCR-TRV50) is used to visualize and record the flow structures, and the exposure time is $1 / 30$ sec. All images, recorded on a cassette tape, are transmitted to a PC for processing and analysis using Corel Video Studio software.

\section{J. Uncertainty Analysis and Experimental Repeatability}

Formulae for evaluating the uncertainty levels here can be found in [19], and the results of uncertainty analyses are shown in Table 1. Figure 4 presents the experimental repeatability of separation angles between front stagnation points and separation points at each $R e$ under $15 \mathrm{~mm}$ diameter cylinder geometry.

TABLE 1

SUMMARY OF UNCERTAINTY ANALYSIS

\begin{tabular}{ll}
\hline \hline Parameters & Uncertainty \\
\hline $\mathrm{D}_{i}, \mathrm{D}_{o}, \mathrm{a}, \mathrm{b}, \mathrm{L}$ & $\pm 0.5 \mathrm{~mm}$ \\
$\mathrm{~A}$ & $\pm 1.27 \%$ \\
$\mathrm{~A}_{s, b}$ & $\pm 3.34 \%$ \\
$\mathrm{~A}_{c, B}(15 \mathrm{~mm}$-diameter cylinder $)$ & $\pm 2.80 \%$ \\
$\mathrm{~A}_{c, B}(20 \mathrm{~mm}$-diameter cylinder $)$ & $\pm 3.56 \%$ \\
$\mathrm{~V}_{0}$ & $\pm 2.2 \%$ \\
$U_{i n}$ & $\pm 2.54 \%$ \\
$V_{w}(15 \mathrm{~mm}$-diameter sphere $)$ & $\pm 3.99 \%$ \\
$V_{w}(15 \mathrm{~mm}$-diameter cylinder $)$ & $\pm 3.56 \%$ \\
$V_{w}(20 \mathrm{~mm}$-diameter cylinder $)$ & $\pm 4.18 \%$ \\
$\operatorname{Re}(15 \mathrm{~mm}$-diameter cylinder/sphere $)$ & $\pm 4.19 \%$ \\
$\operatorname{Re}(20 \mathrm{~mm}$-diameter cylinder $)$ & $\pm 3.57 \%$ \\
$\rho$ & $\pm 0.20 \%$ \\
$\nu$ (Greek letter $)$ & $\pm 0.09 \%$ \\
\hline \hline
\end{tabular}




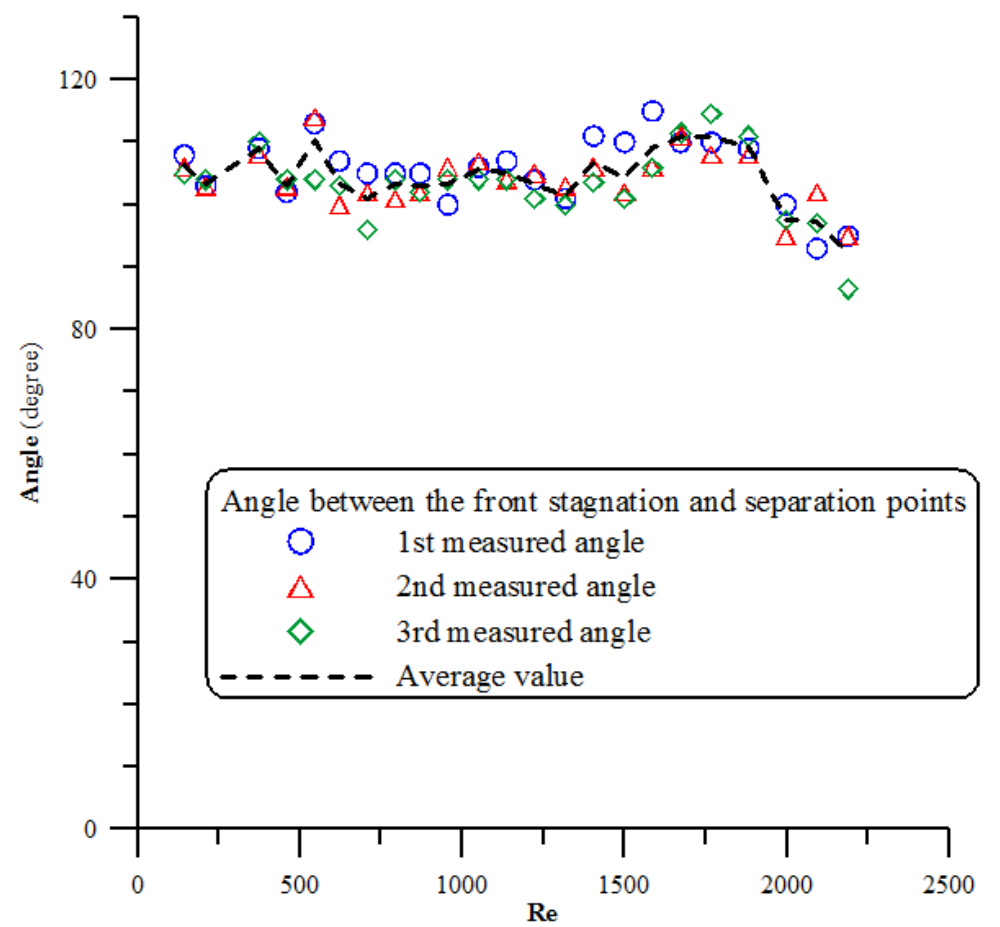

Fig. 4. Diagram of experimental repeatability

For each incoming flow velocity, three measured data are recorded and the corresponding average value, indicated by a dashed curve, is given as well.

\section{RESULTS AND DISCUSSION}

The main subject of the present experiment is to investigate the flow patterns around a cylinder/sphere, which was submitted to blow. The interested parameters are incoming flow velocity $\left(U_{i n}\right)$, air ejection velocity $\left(V_{w}\right)$, and dimension/shape of cylinder/sphere. For the cylinder, two different diameters of cylinder, which are $15 \mathrm{~mm}$ and $20 \mathrm{~mm}$, respectively, are used. Furthermore, the diameter of sphere is $15 \mathrm{~mm}$, and the design of transport line is in horizontal orientation across the stream. The range of $U_{\text {in }}$ is from 0.15 $\mathrm{m} / \mathrm{sec}$ to $2.29 \mathrm{~m} / \mathrm{sec}$. The $V_{w}$ ranges from $1.41 \mathrm{~cm} / \mathrm{sec}$ to $2.64 \mathrm{~cm} / \mathrm{sec}$ for the $15 \mathrm{~mm}$-diameter cylinder, whereas it is from $1.06 \mathrm{~cm} / \mathrm{sec}$ to $1.98 \mathrm{~cm} / \mathrm{sec}$ for the $20 \mathrm{~mm}$-diameter one. As to the sphere, it is ranged from $1.18 \mathrm{~cm} / \mathrm{sec}$ to $7.08 \mathrm{~cm} / \mathrm{sec}$. However, the flow investigations around the cylinder/sphere without blowing are carried out first in the study. The purpose is to confirm the correctness of the experimental results in advance. The descriptions are given in detail as follows:

\section{A. Flows Around and Behind the Cylinder/Sphere with- out Blowing}

1) Flow behaviors for cylinders: The varying parameter for the case of $D=15$ and $20 \mathrm{~mm}$ cylinders is the incoming flow velocity. The Reynolds number, $\operatorname{Re}=(U \times \mathrm{D}) / \nu$, where $\nu$ (Greek letter) is the kinematic viscosity of incoming air at STP condition and is equal to $1.57 \times 10^{-5} \mathrm{~m}^{2} / \mathrm{s}$, is ranged from 143 to 2092 for the $15 \mathrm{~mm}$-diameter cylinder and those are from 191 to 2356 for the $20 \mathrm{~mm}$-diameter one. At $R e=143$, the vortices behind the cylinder are observed. At the beginning of smoke generation, the smoke filaments springing from the separation points of the cylinder are oscillatory. It persists for a few seconds until the downstream smoke rolls back toward the rear of cylinder. As time goes on, two vortices in different sizes present. The large one occupies almost the whole wake behind the cylinder and the small one is observed only in the left or right of far wake. As $R e$ is increased to 210 , smoke filaments show the periodic shedding of vortices. Two different sizes of vortices mentioned at the case of $R e=143$ are also observed, but they become more or less the similar size. However, they are hard to visualize very clearly because of the increase in the incoming flow velocity, which leads the majority of smoke to downstream and only the minority of smoke is recirculated back to the rear of the cylinder. The similar pattern is observed by increasing $R e$ to 372 . The Karman vortex street is shown clearly. Two vortices occupy the wake behind the cylinder alternately. It can be seen that the pattern seems to be disintegrated at downstream of the wake. However, the case of $R e=372$ is near the upper limit for stability by visualization.

At $R e=544$, the disintegrations of flow pattern at down- 
stream of wake occur earlier than that mentioned previously, and the vortices in the wake are vague to identify. They mix up together and become turbulent. Until Re is up to 2092, the flow pattern appears a different type from the previous one. When Re is between 707 and 2092, the Karman vortex street becomes invisible by increasing $R e$ as mentioned previously. For $R e=2092$, it is laminar over the front of the cylinder, then separates downstream, and breaks up into a turbulent wake further downstream.

For the case of $D=15$ and $20 \mathrm{~mm}$-diameter cylinder, the flow patterns for both cases are similar to each other. However, for the $20 \mathrm{~mm}$-diameter cylinder, the critical $R e$, above which the wake breaks up into a turbulent one, is lower than the corresponding $R e$ for $15 \mathrm{~mm}$-diameter cylinder. The vortex street is indistinct, and the wake behind the cylinder is turbulent at $R e=496$. It is due to the rough surface area of the $20 \mathrm{~mm}$-diameter cylinder is larger than the one of the $15 \mathrm{~mm}$-diameter cylinder. The rough surface hastens the wake to become turbulent. Moreover, the flow pattern vibrates on both sides.

2) Flow behaviors for sphere: Unlike two-dimensional flows, such as the flow over a cylinder, the flow over a sphere is considered as a three-dimensional flow, which is more complicated. The flow over a porous sphere is based on $R e=$ 143 to 2092, where $R e$ is based on the sphere diameter and the incoming flow velocity $U_{i n}$, and the kinematic viscosity $\nu$ (Greek letter) of incoming air is the same as the one for the case of cylinder mentioned previously.

At $R e=143$, the wake behind the sphere is steady and symmetric. The vortex-ring is observed at the rear of the sphere. The length of the vortex-ring is approximately equal to diameter of sphere. The front part of vortex-ring is stably attached to the sphere. However, the oscillation is found to exist at the rear of the vortex-ring. According to the previous research conducted by [5], he found that when $R e$ is about 130 , the rear of vortex-ring in the wake behind the sphere begins to oscillate for a very long period. Besides, he also measured the size of the vortex-ring. The observed phenomena in the present work are similar to the findings of [5]. At $R e=210$, the wake behind the sphere is still steady but becomes asymmetric since the vortex-ring shifts away from the axis. Moreover, the smokes from the wake form two parallel threads. The size of vortex-ring is bigger than the one at $R e=143$, and its length is about 1.4D. A similar wake form is obtained as well at $R e=372$. Nevertheless, the two threads become nonparallel. The vortex-ring grows and the length is about $1.8 \mathrm{D}$, and it becomes more and more elongated. From these three measurements, it is found that the size of the vortex-ring is a linear function of $\log R e$, which is found by [5].

The onset of unsteady wake is found at $R e=458$. The double-thread wake becomes unstable and finally the vortex begins shedding from the rear of sphere. The vortexring mentioned above is observed in the wake but it is hard to measure the length of the ring vortex due to the appearance of very slight smoke. The two threads separate and merge alternately along the axis of the sphere. As Re further increases to $621 \sim 2092$, the resultant flow patterns are similar to the one at $R e=458$. At this range of $R e$, the wake behind the sphere becomes more chaotic.

3) Position of separation: The separation angle, measured from the front stagnation point to the separation point, is determined by examining the photos for the cylinder/sphere. Figure 5 illustrates the measurement procedure, and the results are presented in Figure 6.

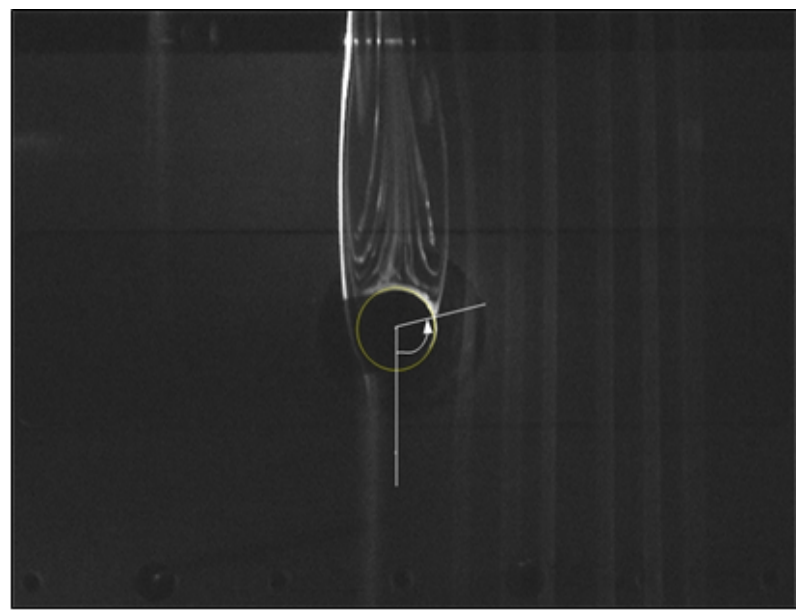

Fig. 5. Definition of separation angle $\theta$

For the case of $15 \mathrm{~mm}$-diameter sphere, the separation angles are ranged from $105^{\circ}$ to $123^{\circ}$. The maximum angle, $123^{\circ}$, is under $R e=143$, whereas the minimum one, $105^{\circ}$, is under $R e=1768$. In the range of $R e=143$ 793, the angle reduces slightly with an increase in $R e$. However, a sharp increase in the separation angle is observed at $R e=870$. Beyond that $R e$, the descendent tendency of the angle with increasing Re appears, even though there are some slight oscillations observed in the range. It is confirmed that the separation point moves forward to the front part of sphere as $R e$ is increased. For the case of cylinder, the unstable oscillation is more apparent than that of the sphere. The separation angles are ranged from $95^{\circ}$ to $111^{\circ}$ for the $15 \mathrm{~mm}$ diameter cylinder and that from $93^{\circ}$ to $116^{\circ}$ for the $20 \mathrm{~mm}$ diameter one. 


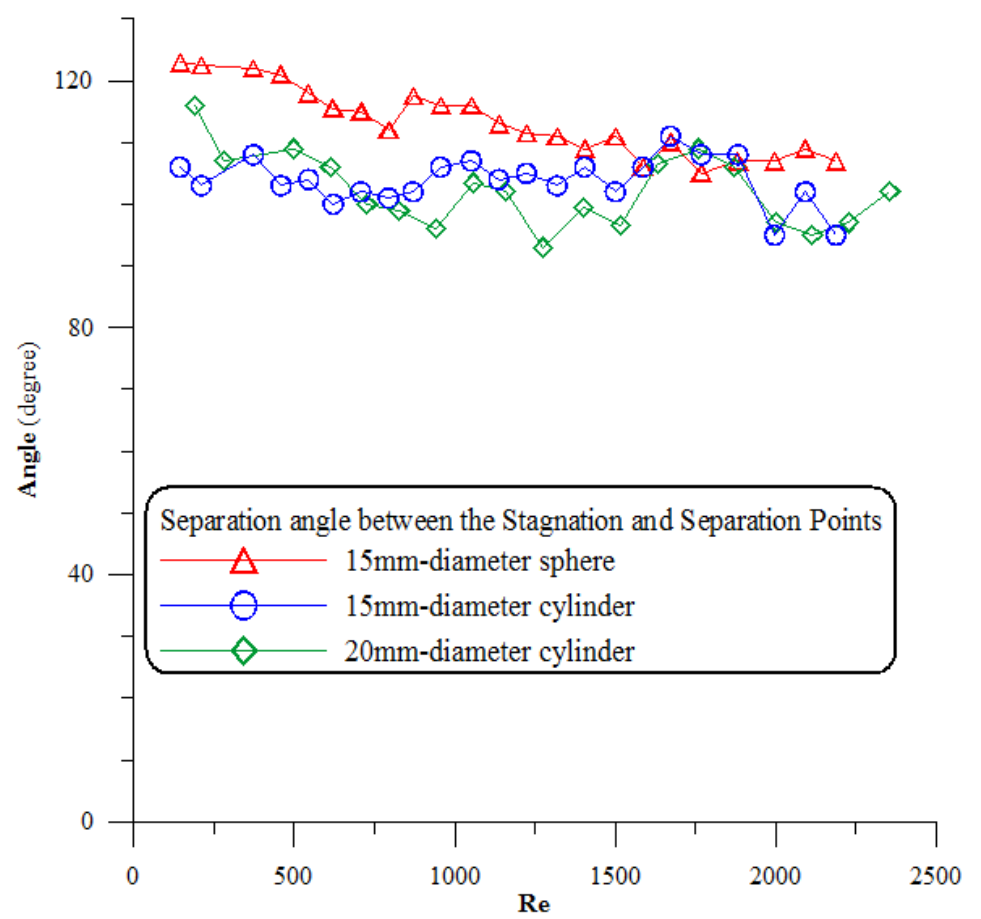

Fig. 6. Separation angle $\theta$ as the function of $R e$

Under the same diameter, the separation occurrence for the cylinder is earlier than that for the sphere one because the flow is subjected to more frictional force in cylinder case. Moreover, the variation of angle with an increase in $R e$ for the large cylinder is more intense than the small one. It may be attributed to the rough surface area which can raise the levels of turbulence. The descendent tendency of the angle with the increasing $R e$ is nonexistent in cylinder case.

\section{B. Flows Around and Behind the Cylinder/Sphere with Blowing}

In order to clarify the effects upon the flow around cylinder/sphere with blowing, several experiments are carried out. Under various $V_{w}$, the resultant flow patterns associated with varying $R e$ are investigated. The flow pattern for each cylinder/sphere with a fixed $V_{w}$ is described in detail as follows:

1) Flow behaviors for cylinders: For the case of $15 \mathrm{~mm}$ diameter cylinder, $V_{w}$ is set at $1.76 \mathrm{~cm} / \mathrm{sec}$ to eject air throughout the whole cylinder surface. At $R e=143$, the wake observed under $V_{w}=1.76 \mathrm{~cm} / \mathrm{sec}$ is different from the one under $V_{w}=0$. The separation points are blown off a distance from the cylinder surface. It looks like the flow around a large cylinder relative to the original one without blowing. The smoke filaments springing from the separation points oscillate slightly. Eventually, a few smokes in downstream are drawn into the wake, which does not connect with the rear of the cylinder. However, the vortices are almost sepa- rated into both sides of wake because of the blowing effect. As $R e$ increases to 210, the periodic vortex shedding shows up. The wake is observed to approach to the rear of cylinder. The separation points are still blown off from the surface of cylinder, but the distance they stand is shorter than that at $R e=143$. The vortices formation in the wake is changed, too. The vortices at $R e=143$ now begin to intermix at $R e$ $=210$, which is due to the great strength of vortices to resist the ejection effect. As $R e=372$ is approaching, the appearance of vortex street becomes obvious. The vortices in the wake form a similar pattern to the one that the cylinder without blowing as mentioned previously. However, it appears more chaotic. The distance between the wake and rear surface of cylinder becomes smaller. When Re is raised, the wake approaches toward rear side of the cylinder. As Re is raised to 907, the wake starts to kiss the surface of cylinder. It seems that the cylinder surface has no air blowing, and this phenomenon is due to the flow field pressure distribution. As the static pressure for the downstream wake is higher than the one caused by air ejection from cylinder, the wake is then pressed back toward the rear side of cylinder. Beyond that, the blow-off wake is no longer obtained as $R e$ is increased further.

For the $20 \mathrm{~mm}$-diameter cylinder, $V_{w}=1.32 \mathrm{~cm} / \mathrm{sec}$ is selected. Figure 7 presents the similar flow behaviors as those at the situation of $D=15 \mathrm{~mm}$ cylinder. In Figure 7(a), as $R e=191$, the flow pattern for the cylinder with blowing is observed. The separation points are not on the cylinder 
surface because of blowing. The smoke filaments originating from the separation points oscillate intensely, and the

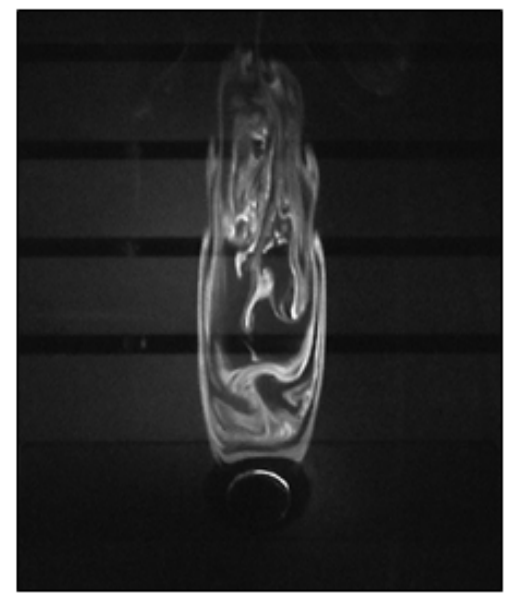

(a) $R e=191$

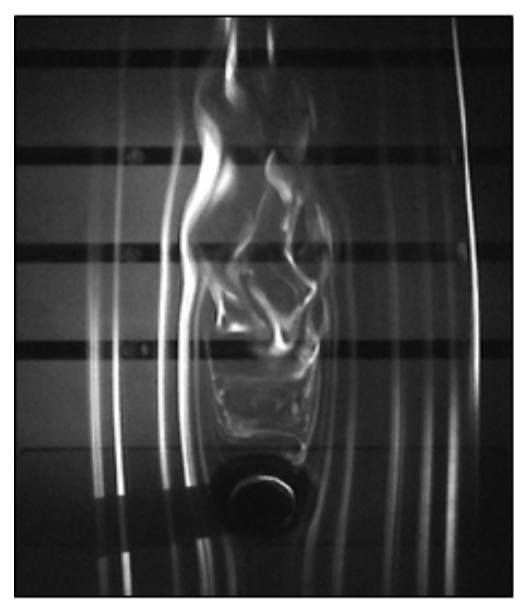

(c) $R e=496$

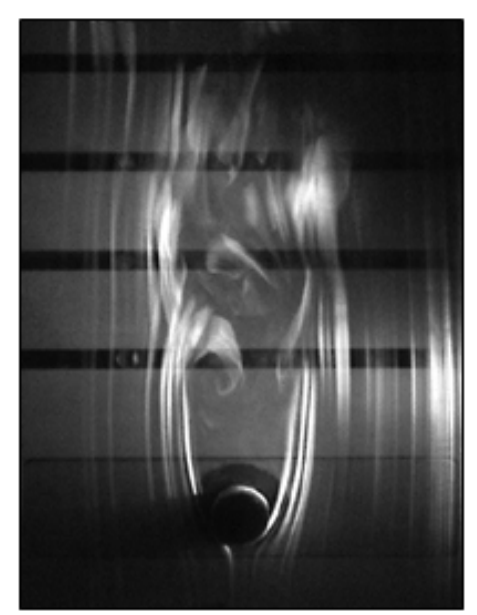

(e) $R e=1057$ downstream smokes are drawn into the wake and form the irregular vortices in the wake.

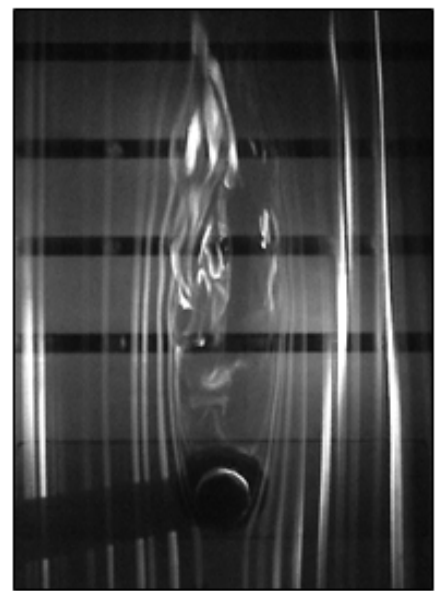

(b) $R e=280$

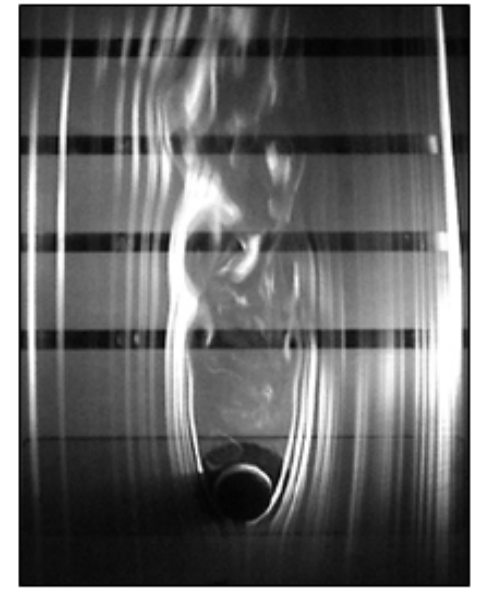

(d) $R e=726$

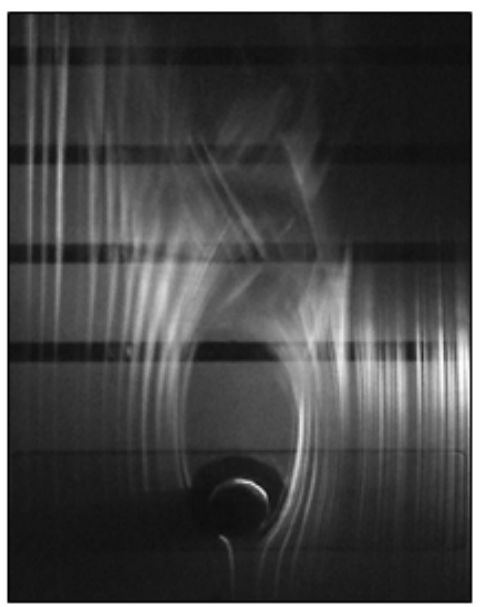

(f) $R e=2000$

Fig. 7. Series of flow behaviors as a function of Reynolds number $(20 \mathrm{~mm}$-diameter cylinder, $V_{w}=1.32 \mathrm{~cm} / \mathrm{sec}$ ) 
The vortices circulate toward the rear of cylinder and finally become almost horizontal at the front part of wake. The wake stands a distance from the rear surface of cylinder. As $R e$ is increased to 280 (see Figure 7(b)), the vortex shedding is observed. The interval between the wake and the rear of cylinder reduces. There are two apparent vortices alternating with each other at the downstream of wake. However, they become chaotic as soon as they approach the border between the wake and the cylinder. It is due to the interference of blowing with the wake. The appearance of vortex street is more evident at $R e=496$, as shown in Figure 7 (c). The wake approaches the rear of cylinder, and the vortices in the wake are turbulent. As Re is increased to 726 (Figure $7(d)$ ), the pattern is disintegrated at the downstream wake. The vortex street seems to be irregular. However, the wake does not attach to the rear of cylinder until $R e$ is 1057, as shown in Figure 7(e). The pattern is similar to the corresponding no blowing case, and the reason is the same as that mentioned previously. Comparing the case of air ejection from cylinder surface with the case of no ejection, it can be seen that the onset of $R e$, above which vortex street is observed, in the former case (with blowing) is greater than the one in the latter. The onset of Re in Figure 7 is 280, whereas corresponding $R e$ is no more than 191 under no blowing condition.

2) Flow behaviors for sphere: Regard to the case of sphere, $V_{w}=4.72 \mathrm{~cm} / \mathrm{sec}$ is selected. At $R e=143$, the wake behind the sphere is shown. At the beginning of smoke generation, the smoke filaments spring from the separation points, which are blown off a height from the surface of sphere. As time goes on, the downstream smokes are drawn into the wake and form the symmetric vortex-ring. However, unlike the vortex-ring mentioned previously which the cylinder/sphere is without blowing, the front part of vortexring is not attached to the rear of sphere. Due to the effect of blowing, the symmetric vortex-ring is separated and shown a " $\Lambda$ " shape, and the rear part of vortex-ring oscillates slightly. As Re reaches 210, the wake is still symmetric. The front parts of vortex-ring are close to the rear of sphere but are not attached either. The " $\Lambda$ " shape vortexring is not obtained and the vortex-ring seems not to be affected by the blowing. Two threads are formed with smokes from the wake and they begin shedding. At $R e=372$, the asymmetric wake is observed, and the vortex-ring shifts off the axis. The wake is still not connected with the rear surface of sphere. Similar pattern is also observed at $R e=458$. The wake is chaotic; however, no vortex-ring is found but the vortex street is obvious. The wake is not attached to the sphere until $R e=1720$. The flow pattern is similar to the one mentioned previously which the sphere is without blowing. 3) Critical $U_{\text {in }}$ for Cylinder/Sphere: Several $V_{w}$ are selected to carry out the same experiments as above for the cylinder/sphere. The critical $U_{i n}$, above which the wake starts to return to the rear of cylinder/sphere, as a function of $V_{w}$ is shown in Figure 8. From Figure 8, we can obtain three correlations for $15 \mathrm{~mm}$-diameter cylinder, 20 $\mathrm{mm}$-diameter cylinder, and $15 \mathrm{~mm}$-diameter sphere, respectively. For the case of $15 \mathrm{~mm}$-diameter cylinder, the correlation between critical $U_{i n}$ and $V_{w}$ is as follows:

$$
U_{\text {in }}=-1.8886+1.6119 \times V_{w}
$$

Similarly, the correlation for $20 \mathrm{~mm}$-diameter cylinder is:

$$
U_{\text {in }}=-1.4299+1.7036 \times V_{w}
$$

The case for the $15 \mathrm{~mm}$-diameter sphere is also dealt with the same manner and the correlation is:

$$
U_{\text {in }}=-0.1981+0.4459 \times V_{w}
$$

The results in Figure 8 manifest that the critical $U_{i n}$ is almost linearly varied with $V_{w}$ no matter what the shape/dimension of cylinder/sphere is. However, it is apparent that the trend of critical $U_{i n}$ under $V_{w}>1.5 \mathrm{~cm} / \mathrm{sec}$ exhibits the following rule: Critical $U_{\text {in }}$ of $D=20 \mathrm{~mm}$ cylinder $>$ Critical $U_{\text {in }}$ of $D=15 \mathrm{~mm}$ cylinder $>$ Critical $U_{\text {in }}$ of $D=15 \mathrm{~mm}$ sphere.

It may be attributed to that under a fixed $V_{w}$, the ejection volumetric flow rate of $20 \mathrm{~mm}$-diameter cylinder is greater than the others because of its greater surface area. Large ejection rate leads to a large static pressure around the surface of cylinder. It also can be seen that the slopes of Equation 1 and 2 are almost the same. Therefore, a region map is redrawn in Figure 9 in terms of $V_{w}(D / L)$ and $U_{i n}(D / L)^{0.5}$ as two parameters, where $D$ and $L$ are diameter and length of the cylinder, respectively. From Figure 9, the critical $U_{i n}$ and its corresponding $V_{w}$ of $D=15$ and $20 \mathrm{~mm}$ cylinder can derive a dimensionless correlation between $V_{w}(D / L)$ and $U_{\text {in }}(D / L)^{0.5}$. Furthermore, this correlation is independent of the diameter of cylinder, and can be expressed as follows:

$$
U_{i n} \times(D / L)^{0.5}=-0.1549+1.6447 \times\left[V_{w} \times(D / L)\right]^{2}
$$

The application of the above equation is discussed in detail later. 


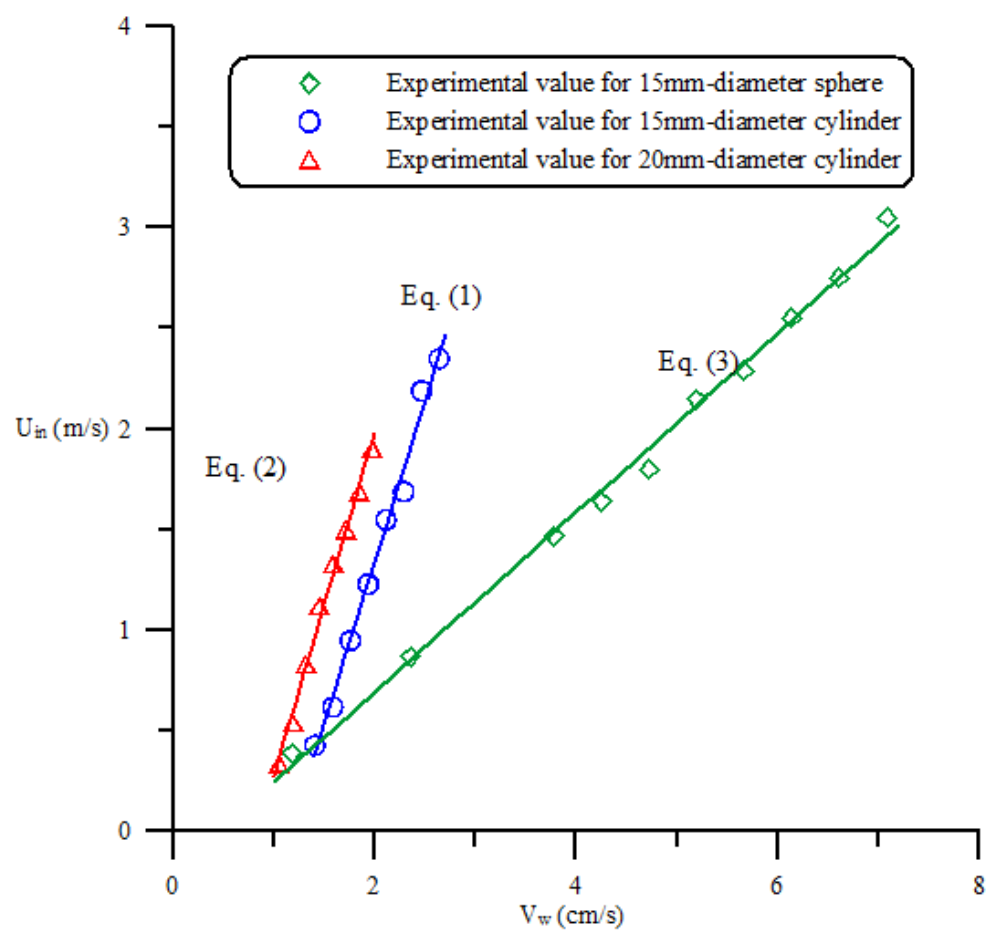

Fig. 8. Critical $U_{i n}$ as a function of $V_{w}$

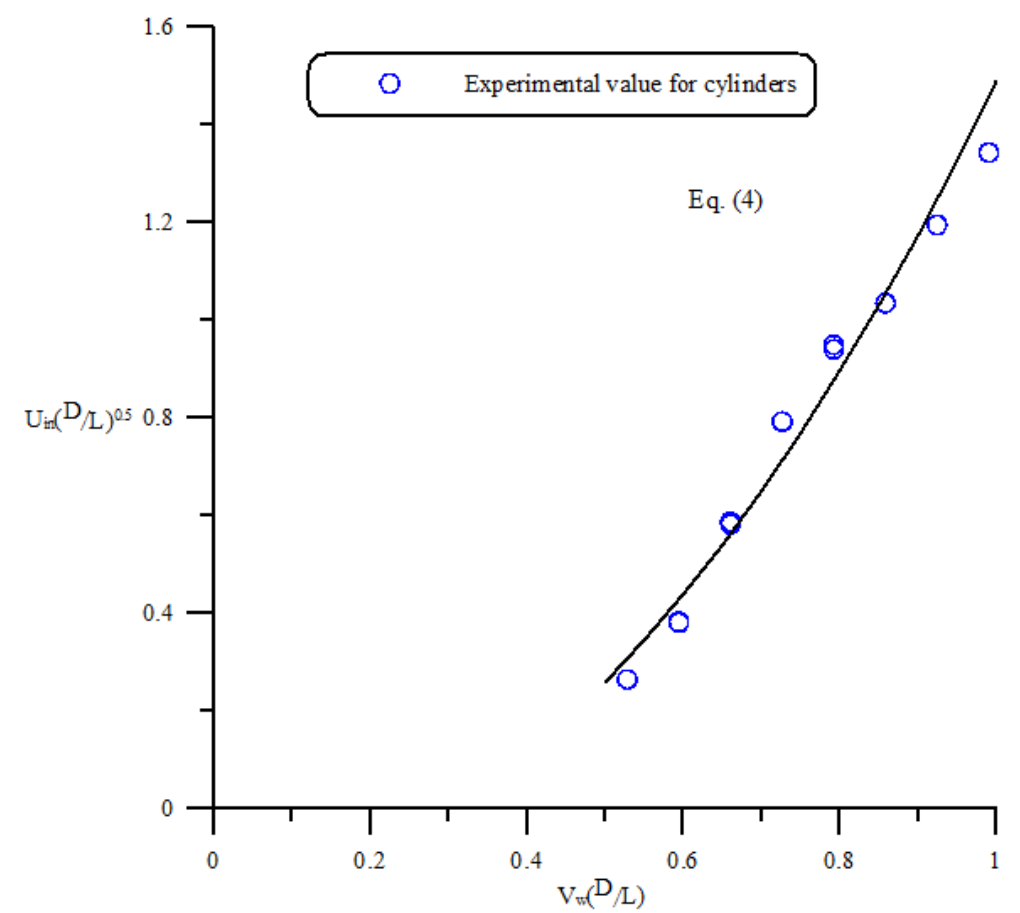

Fig. 9. Dimensionless critical $U_{i n}$ and its corresponding dimensionless $V_{w}$

\section{Comparisons with Other Studies}

The size of cylinder used in the combustion experiment of [3] is different from the ones used in this study. Therefore, Equation 4 is employed to deduce $V_{w}$ and critical $U_{i n}$ for the size of cylinder in [3]. The diameter of cylinder used in [3] is $30 \mathrm{~mm}$ and its length $=40 \mathrm{~mm}$ is the same as the ones used herein. The results of the deduction are shown in Figure 10. The critical $U_{i n}$, above which the lift-off flame is drawn back to become a wake flame again, and the corresponding $V_{w}$ used by [3] are also shown in Figure 10. The cold flow behaviors can correspond to the resultant flame behaviors mentioned in [3], which the lift-off flame is transformed into second wake flame, with the same Re. In Fig- 
ure 10, for the case of cold flow experiments, the trend for the variation of critical $U_{i n}$ with the increasing $V_{w}$ is almost linear, and the left side of region map infers that the wake returns to the rear of cylinder. However, since the flow in combustion involves many complicated factors as mentioned in [3], it can be seen that $V_{w}$ barely influences the mechanism of lift-off flame transforming into second wake flame. The resultant cold flow visualizations in this study just provide a part of assistance to realize the physical mechanism for the occurrence of the second wake flame in [3]. Besides, [1] found that a large fuel-ejection area of cylinder surface re- sults in a high critical $U_{i n}$ that causes the lift-off flame dropping back to the second wake flame. This can be analogous to this study that the bigger cylinder with the greater critical $U_{i n}$ under a fixed $V_{w}$.

The measurements and visualizations are compared with [14], too. The maximum discrepancy of the flow velocity in the stream-wise direction is within $8 \%$, and the average discrepancy is $4.97 \%$ at $U_{i n}=10 \mathrm{~m} / \mathrm{sec}$. Besides, good agreement in the distribution of flow field behind the circular cylinder is also revealed.

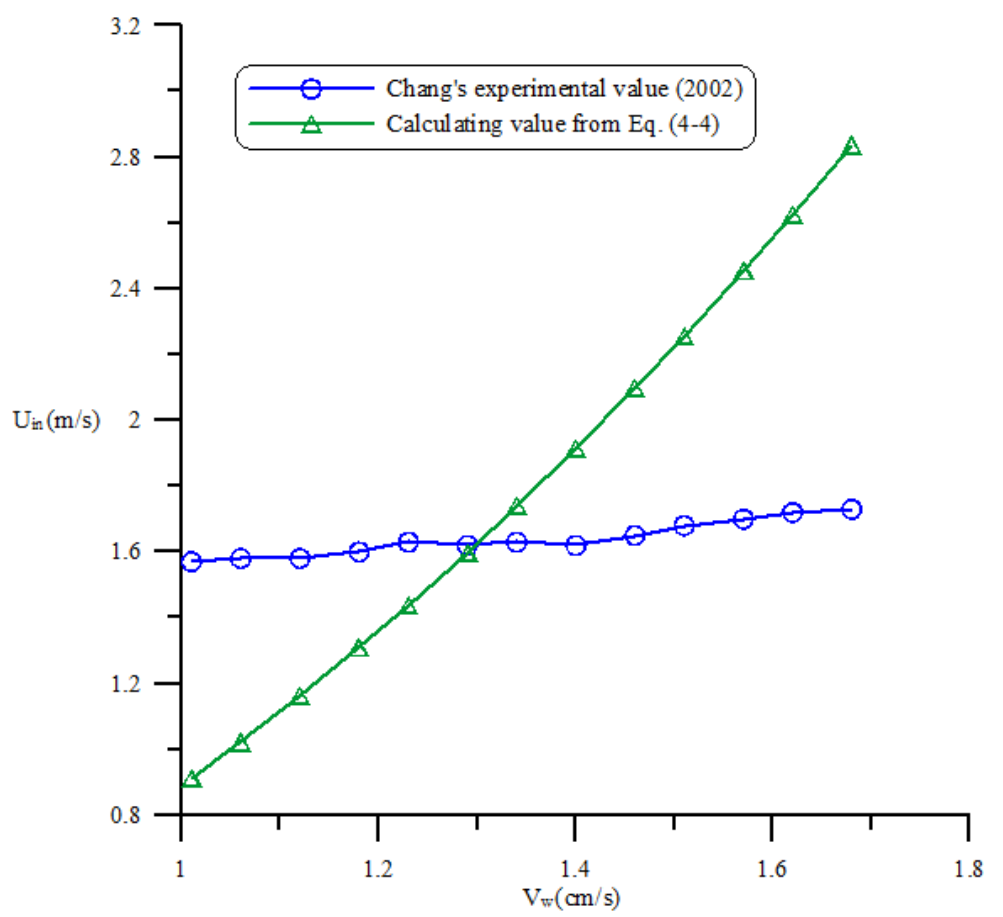

Fig. 10. Comparison with the combustion experiment of [3]

\section{CONCLUSION}

Flow over the cylinder/sphere has been carried out in this study. Wind tunnel, porous circular cylinder, porous sphere, laser-sheet lighting, and smoke-generation wire are adopted in this experimental study. This study is divided into dual parts. First part deals with flow field with no air ejection from the surface of cylinder/sphere, and the second one deals with the flow field for air ejection from cylinder/sphere surface. The goal for the first part is to compare with other studies to identify the correctness of the measurements and visualizations.

For each $V_{w}$, the resultant flow types of cylinder/sphere associated with changing Reynolds number are studied. At $D$ $=15 \mathrm{~mm}$ (for cylinder) and $V_{w}=1.76 \mathrm{~cm} / \mathrm{sec}$, as $R e$ is raised to 907 , the wake starts to kiss the surface of cylinder. It seems that the cylinder surface has no air blowing, and this phenomenon is due to the flow field pressure distribution. As the static pressure for the downstream wake is higher than the one caused by air ejection from cylinder, the wake is then pressed back toward the rear side of cylinder. Furthermore, several $V_{w}$ are selected to carry out the similar experiments, and a correlation between $V_{w}$ and the critical inflow velocity, above which the wake returns toward the rear side of cylinder/sphere, is obtained. The correlation is as follows:

$$
U_{i n}=-1.8886+1.6119 \times V_{w}
$$

For the $20 \mathrm{~mm}$-diameter cylinder with $V_{w}=1.32 \mathrm{~cm} / \mathrm{sec}$, the critical $U_{i n}$ is found at $R e=1057$. The flow behaviors for the $20 \mathrm{~mm}$-diameter cylinder are similar to those for the 15 
mm-diameter one mentioned previously, and the correlation between $V_{w}$ and critical $U_{i n}$ for $20 \mathrm{~mm}$-diameter cylinder is as follows:

$$
U_{\text {in }}=-1.4299+1.7036 \times V_{w}
$$

According to the critical $U_{i n}$ and its corresponding $V_{w}$ of $D=$ 15 and $20 \mathrm{~mm}$ cylinder, we can derive a dimensionless correlation between $V_{w}(D / L)$ and $U_{i n}(D / L)^{0.5}$. Besides, this correlation is independent of the diameter of cylinder, and can be depicted as follows:

$$
U_{\text {in }} \times(D / L)^{0.5}=-0.1549+1.6447 \times\left[V_{w} \times(D / L)\right]^{2}
$$

The phenomenon of wake returning to the rear of sphere as $R e$ increased is also observed. At $V_{w}=4.72 \mathrm{~cm} / \mathrm{sec}$, the wake is not attached to the sphere until $R e=1720$. Similarly, the correlation between $V_{w}$ and the critical $U_{i n}$ for the $15 \mathrm{~mm}$-diameter sphere is as follows:

$$
U_{\text {in }}=-0.1981+0.4459 \times V_{w}
$$

\section{LIMITATIONS AND FUTURE WORK}

Due to the fund limitation, the smoke-wire technique is used to visualize the flow field. Thus, the quality of image is not good enough, so the use of Particle Image Velocimetry (PIV) is necessary for future detailed flow visualizations.

\section{ACKNOWLEDGEMENT}

The authors would like to thank Global Illuminators for reviewing and correcting this research. Grammatical and writing style errors in the original version have been corrected by Global Illuminators, too.

\section{REFERENCES}

[1] D.-D. Chen, S.-S. Tsa, and C.-H. Chen, "Interaction between counterflow flames over binary Tsuji burners arranged in side-by-side," Combustion Science and Technology, vol. 177, no. 10, pp. 1961-1995, 2005. doi: 10.1080/ 00102200590970348

[2] P. Ocal and K. Pihtili, "Numerical investigation of the flow structure around a cylinder for different fluids and reynolds numbers," International Journal of Applied and Physical Sciences, vol. 3, no. 3, pp. 60-67, 2017. doi: 10.20469/ijaps.3. 50002-3

[3] D. Chen, C. Chang, and C. Chen, "Experimental visualizations of counter flow flame behind porous cylinder," Journal Chinese Society of Mechanical Engineers, vol. 27, no. 3, pp. 321-330, 2006.

[4] G. Rui and Z. Zhaowei, "Forecasting the air passenger volume in Singapore: An evaluation of time series models," International Journal of Technology and Engineering Studies, vol. 3, no. 3, pp. 117-123, 2017. doi: 10.20469/ijtes.3. 40004-3

[5] S. Taneda, "Experimental investigation of the wake behind a sphere at low Reynolds numbers," Journal of the Physical Society of Japan, vol. 11, no. 10, pp. 1104-1108, 1956. doi: 10.1143/jpsj.11.1104

[6] R. Natarajan and A. Acrivos, "The instability of the steady flow past spheres and disks," Journal of Fluid Mechanics, vol. 254, pp. 323-344, 1993. doi: 10.1017/s0022112093002150

[7] E. Achenbach, "Vortex shedding from spheres," Journal of Fluid Mechanics, vol. 62, no. 2, pp. 209-221, 1974. doi: 10. $1017 / \mathrm{s} 0022112074000644$

[8] H. Sakamoto and H. Haniu, "A study on vortex shedding from spheres in a uniform flow," Journal of Fluids Engineering, vol. 112, no. 4, pp. 386-392, 1990. doi: 10.1115/1.2909415

[9] A. Tomboulides, S. Orszag, and G. Karniadakis, "Direct and large-eddy simulations of axisymmetric wakes," in 31st Aerospace Sciences Meeting, Reno, NV, 1993.

[10] T. Johnson and V. Patel, "Flow past a sphere up to a reynolds number of 300," Journal of Fluid Mechanics, vol. 378, no. 34, pp. 19-70, 1999. doi: 10.1017/s0022112098003206

[11] H. Deylami, N. Amanifard, S. Hosseininezhad, and F. Dolati, "Numerical investigation of the wake flow control past a circular cylinder with electrohydrodynamic actuator," European Journal of Mechanics-B/Fluids, vol. 66, no. 4, pp. 71-80, 2017. doi: 10.1016/j.euromechflu.2017.06.002

[12] G. Balaji, S. N. Pillai, and C. S. Kumar, "Wind tunnel investigation of downstream wake characteristics on circular cylinder with various taper ratios," Journal of Applied Fluid Mechanics, vol. 10, no. 3, pp. 69-77, 2017.

[13] K. Javadi, F. Kiani, and M. Tahaye Abadi, "Utilising flags to reduce drag around a short finite circular cylinder," Journal of Turbulence, vol. 19, no. 3, pp. 229-251, 2018. doi: 10.1080/14685248.2017.1414225 
[14] S. Wang, Y. Chen, and Y. Z. Liu, "Measurement of unsteady flow structures in a low-speed wind tunnel using continuous wave laser-based TR-PIV: Near wake behind a circular cylinder," Journal of Visualization, vol. 21, no. 1, pp. 73-93, 2018. doi: $10.1007 / \mathrm{s} 12650-017-0445-3$

[15] P. Meliga, "Computing the sensitivity of drag and lift in flow past a circular cylinder: Time-stepping versus selfconsistent analysis," Physical Review Fluids, vol. 2, no. 7, pp. 73-95, 2017. doi: 10.1103/physrevfluids.2.073905

[16] C. Demartino and F. Ricciardelli, "Aerodynamics of nominally circular cylinders: A review of experimental results for civil engineering applications," Engineering Structures, vol. 137, no. 34, pp. 76-114, 2017. doi: 10.1016/j.engstruct. 2017.01.023

[17] D. L. Gao, W. L. Chen, H. Li, and H. Hu, "Flow around a circular cylinder with slit," Experimental Thermal and Fluid Science, vol. 82, pp. 287-301, 2017. doi: 10.1016/j.expthermflusci.2016.11.025

[18] J. C. Yang, M. K. Donnelly, N. C. Prive, and W. L. Grosshandler, "Dispersed liquid agent fire suppression screen apparatus," National Instituite of Standards and Technology Gaithersburg MD Building and Fire Research Lab, Gaithersburg, MD, Tech. Rep., 1999.

[19] R. Fox and A. T. McDonald, Introduction to Fluid Mechanics. Toronto, Canada: John Wiley and Sons, 1994. 